\title{
Analysis of EEG signal of specific epileptic patient prior to its occurrence
}

\author{
Sachin Shrestha*, Rupesh Dahi Shrestha ${ }^{+}$, Amit Shah $^{\#}$, Bhojraj Thapa** \\ Department of Electronics and Communication Engineering \\ Nepal Engineering College, Changunarayan, Bhaktapur, Nepal \\ *shr34.sachin@gmail.com, ${ }^{+}$rupeshd.shrestha@gmail.com, \\ "1979.amit.shah@gmail.com, **anzanthapa@gmail.com
}

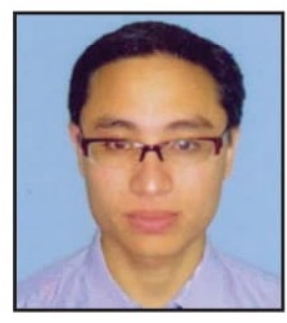

Sachin Shrestha has completed his Masters in Information and Communication Engineering from IOE, Pulchowk Campus. He has been involved in teaching profession in for last 11 years and is currently working as Assistant Professor at Nepal Engineering College, Changunarayan, Nepal. His research interest is ultra-low powered system design, biomedical signal processing, embedded system design and image processing.

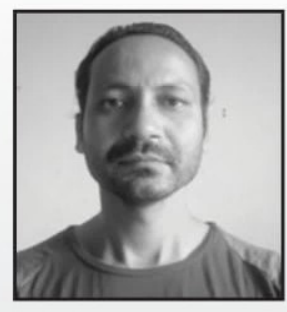

Amit Shah has completed his Masters in Information and Communication Engineering from IOE, Pulchowk Campus. He has been involved in teaching profession in engineering colleges for 12 years, currently working as Assistant Professor in nec, Changunarayan, Nepal. His research interest is bio-signal processing and speech processing.

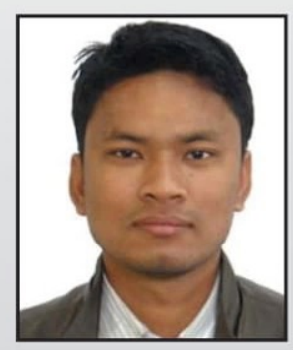

Rupesh Dahi Shrestha received the $B E$ degree in Electronics and Communication from Nepal Engineering College in 2005, and MSc degree in Information and Communication Engineering from Pulchowk Campus in 2013. He has experience of 12 years in teaching and is currently working as Assistant Professor and Head of the Department of Electronics and Communication Engineering, Nepal Engineering College. His research interests include signal processing, robotics and embedded system.

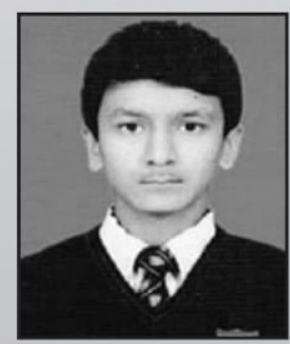

Mr. Bhoj Raj Thapa has been doing his Bachelor's Degree from Nepal Engineering College, Pokhara University. He has done +2 from Liverpool International College under HSEB. He has done projects in his college life related to Android App Development, Signal Processing, and Embedded Systems.

\begin{abstract}
Epilepsy is a neurological disorder of brain and the electroencephalogram (EEG) signals are commonly used to detect the epileptic seizures, the result of abnormal electrical activity in the brain. This paper is focussed on the analysis of EEG signal to detect the presence of the epileptic seizure prior to its occurrence. The result could aid the individual in the initiation of delay sensitive diagnostic, therapeutic and alerting procedures. The methodology involves the multi-resolution analysis (MRA) of the EEG signals of epileptic patient. MRA is carried out using discrete wavelet transform with daubechies 8 as the mother wavelet. For EEG data, the database of MITBIH of one of the patient with 41 different cases is used. The result showed that a unique pattern is observed during the spectral analysis of the signal over different bands with positive predictive value of $100 \%$, negative predictive value of $82.35 \%$ and the overall accuracy of $85.37 \%$. This unique pattern, basically energy burst in two of the bands of the signal can be used as important feature for the early detection of the epileptic seizure. All the results have been simulated within the Matlab environment.
\end{abstract}

Keywords - Electroencephalography, Epileptic seizure, Discrete wavelet transform, Daubechies 8.

\section{Introduction}

Electroencephalography (EEG) is the recording of electrical activity along the scalp. It measures voltage fluctuations resulting from ionic current flow within the neurons of the brain. In clinical context, it refers to the recording of the brain's spontaneous electrical activity over a short period of time, usually 20-40 minutes, as recorded from multiple electrodes placed on the scalp [1]. The diagnostic applications generally focus on the spectral content of EEG, that is, the type of neural oscillations that can be observed in EEG signals. 
The EEG signal contains several spectral components. The amplitude of a human surface EEG signal is in the range of 10 to $100 \mu \mathrm{V}$. The frequency range of the EEG has a fuzzy lower and upper limit, but the most important frequencies from the physiological viewpoint lie in the range of 0.1 to $30 \mathrm{~Hz}$. The standard EEG clinical bands are the delta $(0.1$ to $3.5 \mathrm{~Hz})$, theta (4 to $7.5 \mathrm{~Hz}$ ), alpha (8 to $13 \mathrm{~Hz}$ ), and beta (14 to $30 \mathrm{~Hz}$ ) bands [2]. EEG signals with frequencies greater than $30 \mathrm{~Hz}$ are called gamma waves.

Epilepsy is a chronic neuronal disorder or group of disorders, characterized by the recurrent (two or more) seizures that usually recur unpredictably in the absence of provoking factors. It is linked to an abnormal and excessive discharge from a set of neurons in a specific locus of the brain. This clinical manifestation consists of sudden and transitory abnormal phenomena which may include alterations in consciousness, motor, sensory, autonomic or psychic behaviours. It affects almost all groups of the society. The prevalence and incidence of epilepsy is varying among the countries with heterogeneous figures.

\section{A. Motivation}

About $1 \%$ of people worldwide (65 million) are suffering from epilepsy, [3] and nearly $80 \%$ of cases are in developing countries [4]. Epilepsy becomes more common with the growing age of people [5] [6]. In the developed world, onset of new cases occurs most frequently in infants and the elderly [7] while in the developing world this is in older children and young adults [8], due to differences in the frequency of the underlying causes. It has been observed that about $5-10 \%$ of all people will have an unprovoked seizure by the age of 80 [9], and the chance of experiencing a second seizure is between 40 and $50 \%$ [10]. In many areas of the world those with epilepsy either have their ability to drive restricted or disallowed [11], but most are able to return to driving after a period of time without seizures. Epilepsy cannot be cured, but seizures are controllable with medication in about $70 \%$ of cases [12]. In those whose seizures do not respond to medication, surgery, neuro-stimulation or dietary changes may be considered. Not all cases of epilepsy are lifelong, and a substantial number of people improve to the point that medication is no longer needed.

Automated seizure onset detection will enable the engineering of novel therapeutic and alerting systems to ease the burden of such seizures. There might be therapeutic system capable of detecting and reacting to the onset of a seizure, a local electrical, thermal or neurochemical stimulus that can halt the progression of a seizure prior to the development of clinical symptoms. Moreover, just-in-time, local therapy could relieve patients of the toxic side effects that accompany systemic administration of multi anti-epileptic drugs. An alerting system equipped with seizure onset detection could warn the patient of seizure prior to the development of the debilitating symptoms, or could notify a family member so that the consequences of a seizure can be limited.

\section{B. Spectral analysis of EEG}

As has been mentioned earlier, EEG signals have several spectral components indicating different state of mind. Epilepsy is also indicated with the rise in the energy level of the EEG signal. The multi-resolution analysis of the signal will help in identifying the range of frequencies in which the energy will be higher. The occurrence of epileptic seizure is mostly associated with the higher frequency range usually in the gamma range of EEG. The multi-resolution analysis will help in the effect of the epilepsy in different bands and the one which shows the prominent change before the occurrence of epilepsy can be used as an important feature for further analysis for the onset detection.

\section{Onset detection of epileptic seizure}

Various state of art designs have been implemented to analyse the EEG signals. EEG signals have been used widely to analyse not just the disorders associated with the brain but also with the various states of the brain like the emotions associated with the nervous systems. Research into seizure detection methods began with the development of seizure event detectors [13], the difference between them being the increased accuracy performance with the later. The detectors developed were meant to detect the seizures of any individual with epilepsy i.e. they were patient nonspecific. The variability within EEG severely limited the detection accuracy of such detectors. To improve the performance, investigators developed patient specific event detectors i.e. detectors i.e. detectors that could be tailored to EEG of an individual [14]. Years later, the development of diagnostic and therapeutic applications that require initiation following seizure onset motivated the development of seizure onset detection algorithms. 
One of the earliest patient non-specific seizure event detectors was the one developed by Gotman [13] in 1982. The Gotman algorithm searches for the Hallmark sign of seizures i.e. rhythmic activity. The algorithm sequentially searches a number of EEG channels for the presence of rhythmic activity with a dominant frequency between $3-20 \mathrm{~Hz}$ and an amplitude at least 3 times greater than that of a background window; whenever the degree of rhythmicity exceeds a threshold on at least two channels and persists for 4 seconds a seizure is declared. The Reveal algorithm developed by Wilson [15] decomposes 2 second EEG epochs from each input channel into time-frequency atoms using the Matching Pursuit algorithm. Hand coded and neural network rules are then employed to determine whether features derived from the atoms of a channel are consistent with a seizure taking place on that channel. Saab developed a patient nonspecific seizure onset detector [16] algorithm that uses features derived from a wavelet decomposition of each EEG channel to estimate the probability of a seizure event. Qu developed the first patient specific seizure onset detection algorithm [17] [18] [19] [20]. Shoeb had proposed the machine learning based patient specific seizure onset detector [21]. The detector uses a support vector machine to classify a feature vector that automatically encodes the time evolution of spectral and spatial features within the scalp EEG.

A lot of works has been done on the onset detection of the epilepsy however they are confined to the detection of the epilepsy rather than the effort toward the predictive model for the occurrence of the epilepsy. This paper is focussed more on the feature analysis for the earlier detection of the possible occurrence of epilepsy.

\section{Algorithm Development}

The overall algorithm comprises of basically two major sections. The first one is the extraction of the signal and its pre-processing and the second one is the multi-resolution analysis of the signal.

\section{A. EEG signal extraction and pre-processing}

For this research paper, the database of MIT BIH has been used. The different cases presented in the database has been grouped into 23 cases, collected from 22 subjects ( 5 males, ages 3-22; and 17 females, ages 1.5-19). Here in this paper only one patient out of them has been chosen as specific case for the study purpose. According to the CHB-MIT database [21] [22] [23] [24], first patient is 11 years old girl. The database contains 41 different EEG sample file in total, each of 1 for almost all the files. So, in total it is a continuous record of 41 hours, with breaks in some of them. Out of these 41 cases, there are six cases of epileptic seizure.

The technical details associated with the signal includes, the usage of sampling frequency of $256 \mathrm{~Hz}$, sampling period of 0.00390626 second, duration of 1 hour for most of the data and the 23 leads for the analysis of each case.

\section{B. Discrete wavelet transform of the EEG signal}

The Discrete Wavelet Transform analyses the signal at different frequency bands with different resolutions by decomposing the signal into a coarse approximation and detail information. DWT employs two sets of functions, called scaling functions and wavelet functions, which are associated with low pass and high pass filters, respectively. The decomposition of the signal into different frequency bands is simply obtained by successive high pass and low pass filtering of the time domain signal.

The original signal $x[n]$ is first passed through a half band high pass filter $g[\mathrm{n}]$ and a low pass filter $\mathrm{h}[\mathrm{n}]$. After the filtering, half of the samples can be eliminated according to the Nyquist rule, since the signal now has a highest frequency of pi/2 radians instead of pi, where $2 \mathrm{pi}$ is the sampling frequency, fs in terms of radian. The signal can therefore be subsampled by 2 , simply by discarding every other sample. This constitutes one level of decomposition and can mathematically be expressed as follows:

$$
\begin{aligned}
& \mathrm{Y}_{\text {high }}=g[k] \\
& =\sum_{n=0} x[n] \cdot g[2 k-n] \sum_{n=0} x[n] \cdot g[2 k-n] \ldots(1) \\
& \mathrm{Y}_{\text {low }}=h[k] \\
& =\sum_{n=0} x[n] \cdot h[2 k-n] \sum_{n=0} x[n] \cdot h[2 k-n] \ldots \ldots(2)
\end{aligned}
$$

where, $\mathrm{Y}_{\text {high }}[k]$ and $\mathrm{Y}_{\text {low }}[k]$ are the outputs of the high pass and low pass filters, respectively, after subsampling by 2 .

This decomposition halves the time resolution since only half the number of samples now 
characterizes the entire signal. However, this operation doubles the frequency resolution, since the frequency band of the signal now spans only half the previous frequency band, effectively reducing the uncertainty in the frequency by half. The above procedure, which is also known as the sub-band coding, can be repeated for further decomposition. At every level, the filtering and subsampling will result in half the number of samples (and hence half the time resolution) and half the frequency band spanned (and hence double the frequency resolution).

With the subsampling operator $\downarrow$ (down-sampling), shown in equation (3), equations (1) and (2) can be written more concisely, shown as equations (4) and (5).

$$
\begin{aligned}
& (\mathrm{Y} \downarrow n)[k]=\mathrm{Y}[k n] \\
& \mathrm{Y}_{\text {low }}=(x * g) \downarrow 2 \ldots \\
& \mathrm{Y}_{\text {high }}=(x * h) \downarrow 2
\end{aligned}
$$

\section{Calculation of energy of different bands of EEG}

DWT decomposes original signal into two different frequency bands in its $1^{\text {st }}$ level decomposition. In this research work, the decomposition has been done up to five levels resulting the six different frequency bands. The so obtained frequency bands are:

TABLE I

Frequency bands and associated range of frequency

\begin{tabular}{|c|c|}
\hline Frequency bands & Range of frequency $(\mathbf{H z})$ \\
\hline Band 1 & 0 to 4 \\
\hline Band 2 & 4 to 8 \\
\hline Band 3 & 8 to 16 \\
\hline Band 4 & 16 to 32 \\
\hline Band 5 & 32 to 64 \\
\hline Band 6 & 64 to 128 \\
\hline
\end{tabular}

After obtaining the coefficients for these different frequency bands, the energy associated with each of them is calculated using the formulation based on Parseval's theorem as shown in equation (6).

$$
\begin{aligned}
& E D i=\sum_{j=1}^{N}\left|D_{i j}\right|^{2}, i=1, \\
& E A_{l}=\sum_{j=1}^{N}\left|A_{i j}\right|^{2} \ldots \ldots \ldots .
\end{aligned}
$$

where $i=1, \ldots \ldots, l$ is the wavelet decomposition level from level 1 to level $l$. $\mathrm{N}$ is the number of the coefficients of detail or approximate at each decomposition level. $E D_{i}$ is the energy of the detail at decomposition level $i$ and $E A$, is the energy of the approximate at decomposition level $l$. The value of $l$ is taken 5 . Since the number of coefficients vary with the level, the values obtained have been scaled down by the number of coefficients.

In DWT, it is very important to choose a suitable wavelet alongside the number of levels of decomposition. Here, the Daubechies 8 has been chosen as a mother wavelet for DWT. The reason behind choosing this wavelet for EEG analysis is its smoothing feature which is suitable for detecting changes of the EEG signals. The scaling function and the wavelet function of this mother wavelet are shown in Fig. 1 and Fig. 2. These wavelets are asymmetric in nature and shows bi-orthogonal nature. This wavelet analysis can represent EEG sub-bands as a weighted sum of shifted and scaled version of an original wavelet without losing any information of energy. Since this transformation can retain the frequency as well time properties of the signal, this is considered superior to the other methods like Fourier transform for this research work.

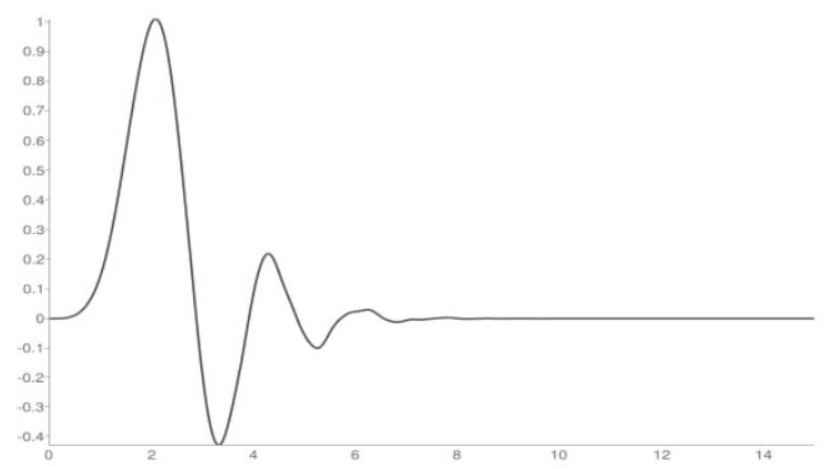

Fig. 1 Scaling function of Daubechies 8 wavelet

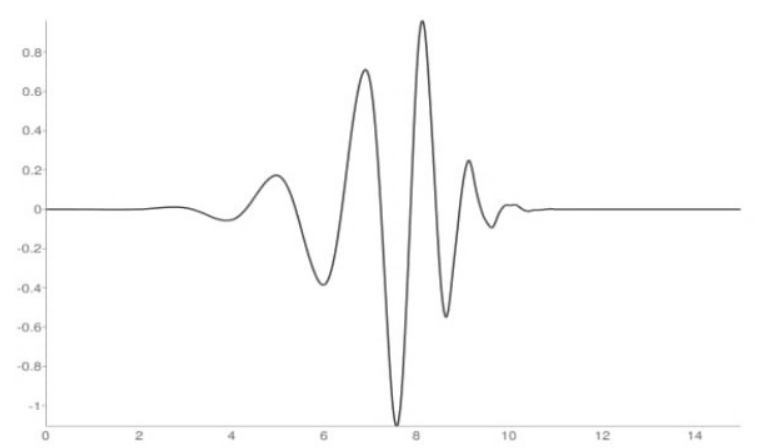

Fig. 2 Wavelet function of Daubechies 8 wavelet 


\section{Windowing and shifting for energy calculation}

For the calculation of the energy of different bands, the calculation is done over a window of 256 samples which corresponds to the duration of 1 second. Next, the window is shifted by 128 samples that corresponds to the duration of 0.5 second. The window so taken is of rectangular nature and this window based calculation helps in obtaining better resolution for time. Next, the shift by $50 \%$ i.e. 128 samples with window size of 256 samples will help in eliminating the edge effect of the window. Thus the period of 1 hour i.e. 3600 seconds will give $6 \times 7199$ values corresponding to the each lead of EEG signal.

\section{Result and Discussion}

As mentioned in earlier section, the paper is focussed on the analysis of only one patient who is an eleven years old girl. The database contains 41 different EEG sample file of 1 hour of EEG samples each, in almost every file, taken from 23 different leads, resulting the data matrix of size $23 \times 921600$ for each file. Out of these 41 files, 7 files show the case of epilepsy, namely, chb01_03, chb01_04, chb01_15, chb01_16, chb01_18, chb01_21, chb01_26.

With the window size of 256 samples and shift of 128 sample, 7199 windows were obtained for signal of duration one hour of samples. DWT implementation on each window gave energy for 6 different frequency bands of size 1 × 6 and for 7199 windows, matrix of size 7199 × 6 was obtained as output for every lead. The EEG signal along with its energy spectrum for 6 different bands are shown in figures (3) to (9).

From the observed pattern of the spectral analysis of EEG of different bands, it can be concluded that the first three bands didn't show specific pattern however for the last three bands, a rise in energy is seen earlier to the occurrence of the epileptic seizure. This distinct energy burst could be the one of the features for the early prediction of the occurrence of the epilepsy. Similar observations were seen for the other cases of epilepsy and while testing for the non-epileptic cases, except for few, they weren't present. The details of the results were shown in the Table II.

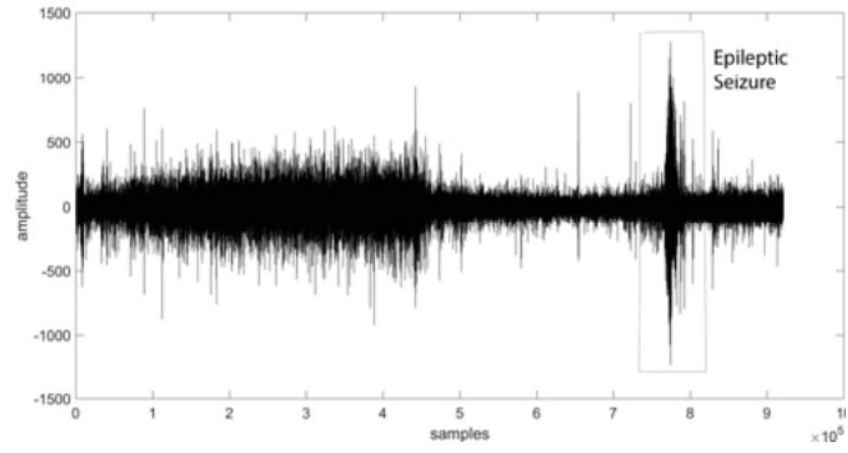

Fig. 3 EEG signal of chb01_03 of lead FP1-F7

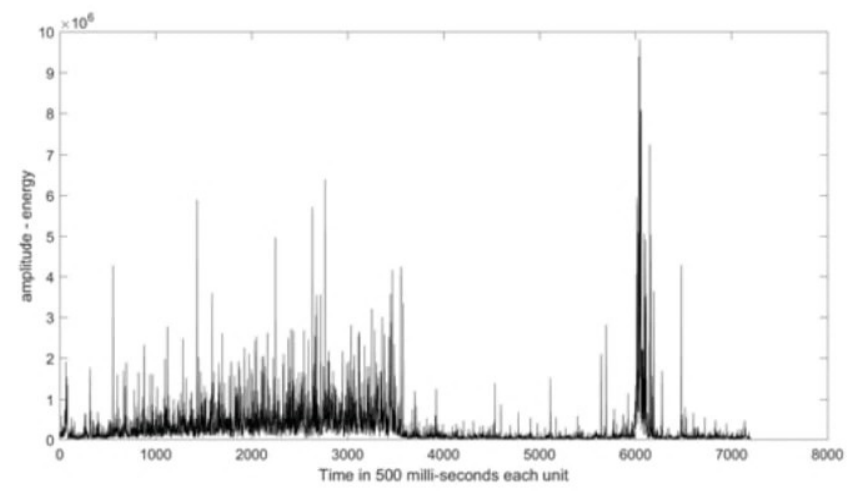

Fig. 4 Energy spectrum of first frequency band (0 to $4 \mathrm{~Hz}$ )

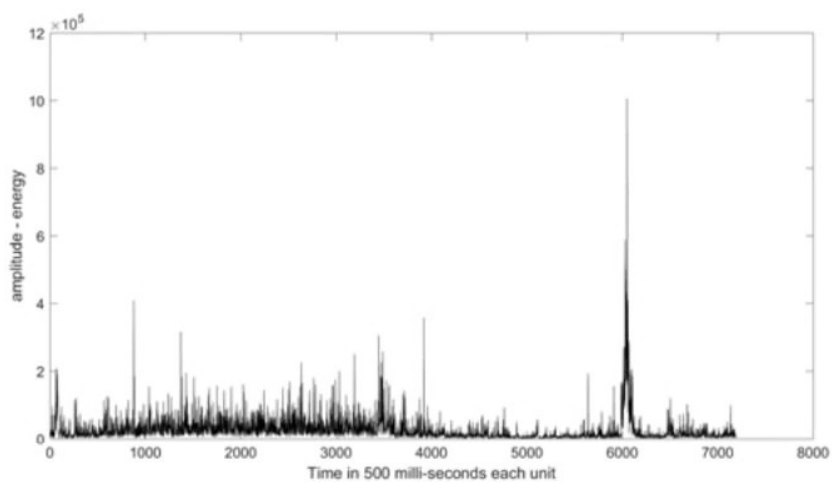

Fig. 5 Energy spectrum of first frequency band (4 to $8 \mathrm{~Hz}$ )

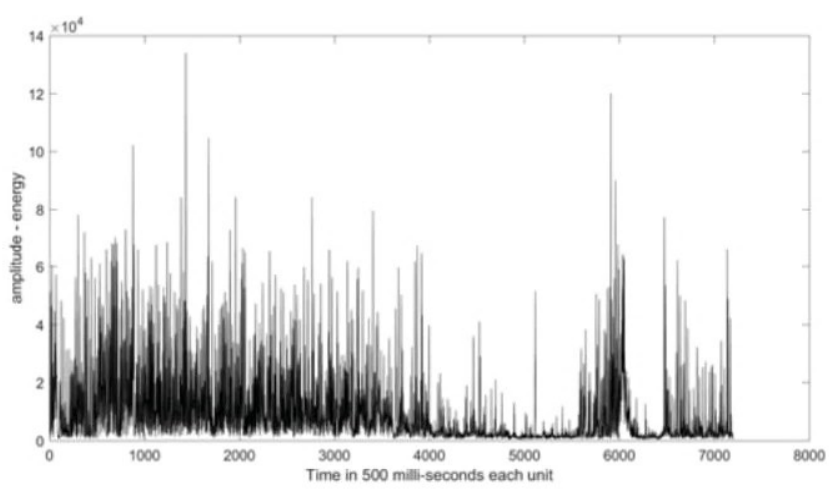

Fig. 6 Energy spectrum of first frequency band (8 to $16 \mathrm{~Hz}$ ) 


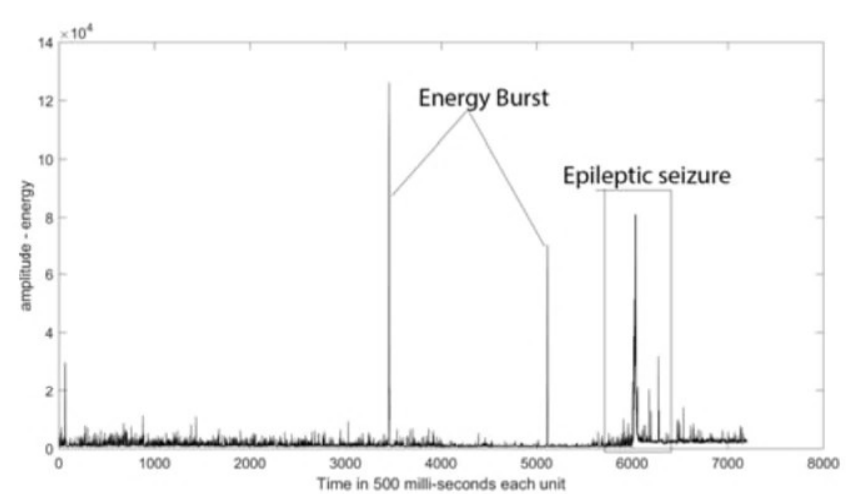

Fig. 7 Energy spectrum of first frequency band (16 to $32 \mathrm{~Hz}$ )

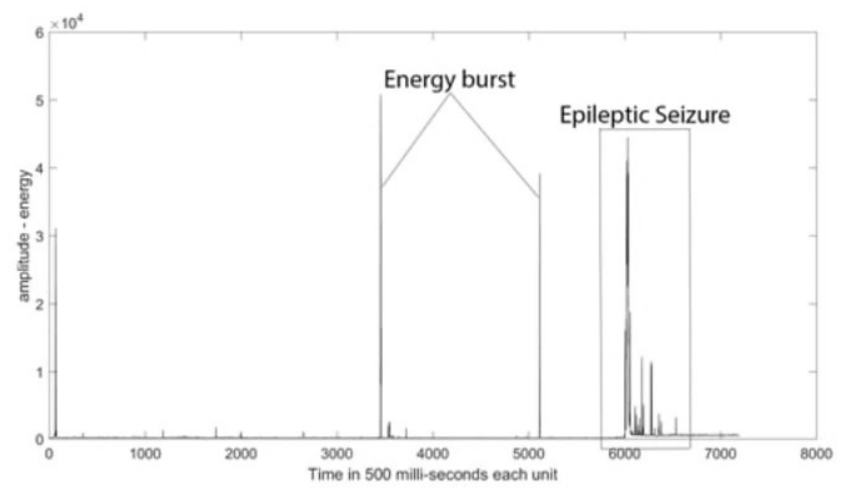

Fig. 8 Energy spectrum of first frequency band (32 to $64 \mathrm{~Hz})$

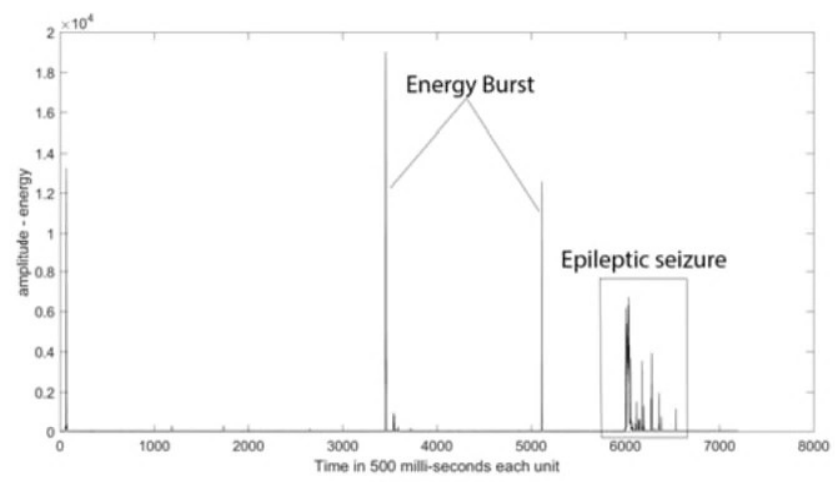

Fig. 9 Energy spectrum of first frequency band (64 to $128 \mathrm{~Hz}$ )

TABLE II

Comparison of the annotations between actual case and observed one based on pattern

\begin{tabular}{|c|c|c|}
\hline Files & $\begin{array}{c}\text { Exact } \\
\text { Annotation }\end{array}$ & $\begin{array}{c}\text { Annotation based on } \\
\text { pattern observed }\end{array}$ \\
\hline Chb01_01 & Absent & Absent \\
\hline Chb01_02 & Absent & Absent \\
\hline Chb01_03 & Present & Present \\
\hline Chb01_04 & Present & Present \\
\hline Chb01_05 & Absent & Absent \\
\hline Chb01_06 & Absent & Absent \\
\hline Chb01_07 & Absent & Absent \\
\hline
\end{tabular}

\begin{tabular}{|c|c|c|}
\hline Files & $\begin{array}{c}\text { Exact } \\
\text { Annotation }\end{array}$ & $\begin{array}{c}\text { Annotation based on } \\
\text { pattern observed }\end{array}$ \\
\hline Chb01_08 & Absent & Absent \\
\hline Chb01_09 & Absent & Absent \\
\hline Chb01_10 & Absent & Absent \\
\hline Chb01_11 & Absent & Absent \\
\hline Chb01_12 & Absent & Absent \\
\hline Chb01_13 & Absent & Absent \\
\hline Chb01_14 & Absent & Absent \\
\hline Chb01_15 & Present & Present \\
\hline Chb01_16 & Present & Present \\
\hline Chb01_17 & Absent & Absent \\
\hline Chb01_18 & Present & Present \\
\hline Chb01_19 & Absent & Absent \\
\hline Chb01_20 & Absent & Absent \\
\hline Chb01_21 & Absent & Absent \\
\hline Chb01_22 & Absent & Absent \\
\hline Chb01_23 & Absent & Absent \\
\hline Chb01_24 & Absent & Absent \\
\hline Chb01_25 & Absent & Absent \\
\hline Chb01_26 & Present & Present \\
\hline Chb01_27 & Absent & Absent \\
\hline Chb01_29 & Absent & Absent \\
\hline Chb01_30 & Absent & Absent \\
\hline Chb01_31 & Absent & Absent \\
\hline Chb01_32 & Absent & Absent \\
\hline Chb01_33 & Absent & Absent \\
\hline Chb01_34 & Absent & Absent \\
\hline Chb01_36 & Absent & Present \\
\hline Chb01_38 & Absent & Present \\
\hline Chb01_39 & Absent & Present \\
\hline Chb01_40 & Absent & Present \\
\hline Chb01_41 & Absent & Present \\
\hline Chb01_42 & Absent & Absent \\
\hline Chb01_43 & Absent & Present \\
\hline Chb01_46 & Absent & Absent \\
\hline
\end{tabular}

\section{Evaluation Procedure}

For the analysis of the result, positive predictive value (PPV), negative predictive value (NPV) and accuracy were calculated. The positive predictive value (PPV) is defined as the ratio of number of true positive (TP) by the summation of number of true positive and number of false positive (FP). A "true positive" is the event that the test makes a positive prediction, and the subject has a positive result under the gold standard, and a "false positive" is the event that the test makes a positive prediction, and the subject has a negative result under the gold standard. Likewise, the 
negative predictive value (NPV) is defined as the ratio of number of true negative (TN) by the summation of number of true negative and number of false negative (FN). A "true negative" is the event that the test makes a negative prediction, and the subject has a negative result under the gold standard, and a "false negative" is the event that the test makes a negative prediction, and the subject has a positive result under the gold standard. The accuracy is calculated as the ratio of summation of true positive and true negative to the summation of true positive, true negative, false positive and false negative [25].

The analysis of the result for 41 cases for different values of TP, TN, FP and FN is shown in Table III.

TABLE III

Observed values for TP, FP, TN and FN

\begin{tabular}{|c|c|}
\hline Frequency bands & Range of frequency $(\mathbf{H z})$ \\
\hline TP & 7 out of 7 \\
\hline FP & 0 out of 7 \\
\hline TN & 28 out of 34 \\
\hline FN & 6 out of 34 \\
\hline
\end{tabular}

Based on the observed values as shown in Table III, the PPV, NPV and accuracy are calculated as $100 \%$, $82.35 \%$ and $85.37 \%$ respectively.

\section{Conclusion}

This paper discussed the multi-resolution analysis of the EEG signal under different bands for a specific patient prior to the occurrence of the epilepsy. The observation of 41 different cases of the patient has shown a unique pattern for the epileptic case and nonoccurrence for the non-epileptic session with the PPV, NPV and accuracy as discussed in the previous section. Since, the prediction of the occurrence of the epileptic seizure is more crucial to alert the patient or his/her near ones, this finding could be a boon for such and thus can be used a very useful feature for the development of the prediction model for the detection of the onset of the epileptic seizure.

\section{Acknowledgment}

The authors would like to thank the University Grants Commission (UGC) of Nepal for funding this project [26]. Also, the authors are very grateful to the MIT BIH for availing the database of epileptic seizure for free for the research purpose.

\section{References}

[1] Niedermeyer E. and da Silva F.L. (2004). Electroencephalography: Basic Principles, Clinical Applications, and Related Fields, Lippincot Williams \& Wilkins.ISBN 0-78175126-8

[2] S. Tong and N.V.Thacor, Engineering in Medicine \& Biology-Quantitative EEG Analysis Methods and Clinical Applications, Boston/ London: Artech House, 2009.

[3] Thurman, DJ; Beghi, E; Begley, CE; Berg, AT; Buchhalter, JR; Ding, D; Hesdorffer, DC; Hauser, WA; Kazis, L; Kobau, R; Kroner, B; Labiner, D; Liow, K; Logroscino, G; Medina, MT; Newton, CR; Parko, K; Paschal, A; Preux, PM; Sander, JW; Selassie, A; Theodore, W; Tomson, T; Wiebe, S; ILAE Commission on, Epidemiology (September 2011). "Standards for epidemiologic studies and surveillance of epilepsy.”.Epilepsia. 52 Suppl 7: $2-26$.

[4] "Epilepsy". Fact Sheets. World Health Organization. October 2012. Retrieved January 24, 2013.

[5] Brodie, MJ; Elder, AT, Kwan, P (November 2009). "Epilepsy in later life". Lancet neurology 8 (11): 1019-30.

[6] Holmes, Thomas R. Browne, Gregory L. (2008). Handbook of epilepsy (4th ed.). Philadelphia: Lippincott Williams \& Wilkins. p. 7.

[7] Wyllie's treatment of epilepsy: principles and practice. (5th ed.). Philadelphia: Wolters Kluwer/ Lippincott Williams \& Wilkins. 2010. ISBN 9781-58255-937-7.

[8] Newton, CR (29 September 2012). "Epilepsy in poor regions of the world.". Lancet 380 (9848): 1193-201.

[9] Wilden, JA; Cohen-Gadol, AA (15 August 2012). "Evaluation of first nonfebrile seizures.". American family physician 86 (4): 334-40.

[10] Berg, AT (2008). "Risk of recurrence after a first unprovoked seizure”. Epilepsia. 49 Suppl 1: 13-8.

[11] L Devlin, A; Odell, M; L Charlton, J. Koppel, S (December 2012). "Epilepsy and driving: current 
status of research" Epilepsy research 102 (3): 13552.

[12] Eadie, MJ (December 2012), "Shortcomings in the current treatment of epilepsy", Expert Review of Neurotherapeutics 12 (12): 1419-27.

[13] J. Gotman, Automatic recognition of epileptic seizure in EEG, Electroencephalograpy and Clinical Neurophysiology, 54:530-540, 1982.

[14] Hao QU and Jean Gotman, Improvement in seizure detection performance by automatic adaptation to the EEG of each patient, Electroencephalography and Clinical Neurophysiology, 86:79-87, 1993.

[15] Scott Wilson, Mark Scheuer, Ronald Emerson, and Andrew Gabor, Seizure detection: Evaluation of the reveal algorithm, Clinical Neurophysiology, 10:2280-2291, 2004.

[16] M.E. Saab and J. Gotman, A system to detect the onset of epileptic seizures in scalp EEG, Clinical Neurophysiology, 116: 427-442, 2005.

[17] Hao Qu, Self adapting algorithms for seizure detection during EEG monitoring, $\mathrm{PhD}$ dissertation, McGill University, 1995.

[18] Hao Qu and Jean Gotman, Improvement in seizure detection performance by automatic adaptation to the EEG of each patient, Electroencephalography and clinical neurophysiology, 86:79-87, 1993.

[19] Hao Qu and Jean Gotman, A seizure warning system for long term epilepsy monitoring, Electroencephalography and clinical neurophysiology, 45:2250-2254, 1995.
[20] Hao Qu and Jean Gotman, A patient specific algorithm for the detection of seizure onset in long term EEG monitoring, IEEE transactions on biomedical engineering, 44:115-122, 1997.

[21] Ali Shoeb, "Application of Machine Learning to Epileptic Seizure Onset Detection and Treatment", PhD Thesis, Massachusetts Institute of Technology, September 2009.

[22] CHB MIT Scalp EEG database, Online: http:// physionet.org/physiobank/database/chbmit, access date: May 2018.

[23] Ali Shoeb, John Guttag, 27th International Conference on Machine Learning (ICML), June 21-24, 2010, Haifa, Israel.

[24] Ali Shoeb, Herman Edwards, Jack Connolly, Blaise Bourgeois, S. Ted Treves, John Guttag, "Patient-Specific Seizure Onset Detection" Epilepsy and Behavior. August 2004, 5(4): 483498. [doi:10.1016/j.yebeh.2004.05.005]

[25] Wikipedia, Positive and Negative predictive values, Online: https://en.wikipedia.org/wiki/ Positive_and_negative_predictive_values, access date: May 2018 .

[26] University Grant Commission, Online: http:// www.ugcnepal.edu.np, access date: May 2018.

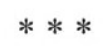

